\title{
Transverse Spin and Momentum in Two-Wave Interference
}

\author{
Aleksandr Y. Bekshaev, ${ }^{1,2}$ Konstantin Y. Bliokh, ${ }^{1,3}$ and Franco Nori ${ }^{1,4}$ \\ ${ }^{1}$ Center for Emergent Matter Science, RIKEN, Wako-shi, Saitama 351-0198, Japan \\ ${ }^{2}$ I. I. Mechnikov National University, Dvorianska 2, Odessa, 65082, Ukraine \\ ${ }^{3}$ iTHES Research Group, RIKEN, Wako-shi, Saitama 351-0198, Japan \\ ${ }^{4}$ Physics Department, University of Michigan, Ann Arbor, Michigan 48109-1040, USA
}

(Received 15 September 2014; published 30 March 2015)

\begin{abstract}
We analyze the interference field formed by two electromagnetic plane waves (with the same frequency but different wave vectors), and find that such a field reveals a rich and highly nontrivial structure of the local momentum and spin densities. Despite the seemingly planar and extensively studied character of the two-wave system, we find that it possesses a transverse (out-of-plane) helicity-independent spin density and also a transverse polarization-dependent momentum density with unusual physical properties. The polarization-dependent transverse momentum represents the so-called Belinfante spin momentum, which does not exert the usual optical pressure and is considered as "virtual" in field theory. We perform analytical estimations and exact numerical simulations of the interaction of the two-wave field with probe Mie particles. The results of these calculations clearly indicate the straightforward detectability of the unusual spin and momentum properties in the two-wave field and strongly motivate their future experimental verifications.
\end{abstract}

DOI: 10.1103/PhysRevX.5.011039

\section{INTRODUCTION}

It is well known, since the seminal works by Poynting [1], that light carries momentum and angular momentum (AM) $[2,3]$. Typical plane-wave or Gaussian-beam states exhibit longitudinal momentum associated with the wave vector $\mathbf{k}$ and also longitudinal (k-directed) spin AM associated with the degree of circular polarization (helicity) $\sigma$. This is in accordance with the "naive" but intuitively clear picture of photons as particles carrying momentum and spin. However, local momentum and angularmomentum densities in structured (i.e., non-plane-wave) optical fields can demonstrate unusual features, which have recently attracted considerable attention. These are "supermomentum" with values higher than $\hbar k$ per photon [4-8], transverse (i.e., orthogonal to k) helicity-independent spin AM [9-13], and transverse helicity-dependent momentum $[10,14,15]$.

Optical momentum and AM are the main dynamical properties of light, which manifest themselves and play a crucial role in various light-matter interactions [16], including laser cooling [17], optical manipulation of small particles [18], and optomechanical systems [19]. Importantly, momentum and AM of light can be transferred to small absorbing particles or atoms [4,7,8,10,17,18,20-22],

Published by the American Physical Society under the terms of the Creative Commons Attribution 3.0 License. Further distribution of this work must maintain attribution to the author(s) and the published article's title, journal citation, and DOI.
Subject Areas: Optics, Photonics, Quantum Physics

generating a radiation-pressure force and torque on the particle [10,23-27]. In other words, the local optical momentum and spin densities can be measured via the translational and spinning motion of the probe particles. This was used for the detection of the above-mentioned extraordinary spin and momentum properties in structured fields $[4,5,7,8,10-13]$.

In this work, we are interested in the transverse momentum and spin AM [9-15]. Thus far, these unusual quantities have been noticed only in evanescent waves, i.e., inhomogeneous near fields defined in half-space and strongly localized in the vicinity of sharp interfaces. An important question is whether the transverse momentum and spin properties can be observed in freely propagating far fields in vacuum, e.g., in usual paraxial laser beams. Here, we find that the simplest propagating non-plane-wave fieldtwo interfering plane waves-also exhibits these extraordinary spin and momentum properties. Despite the seemingly planar and thoroughly studied character of the two-wave system, we discover that such a field possesses a transverse (i.e., out of the plane formed by the two wave vectors, see Fig. 1) helicity-independent spin density and also a transverse polarization-dependent momentum with nontrivial physical properties.

The transverse ( $y$ directed in our geometry) quantities are not restricted by the planar $(x, z)$ wave vector configuration because they are determined by the internal polarization degrees of freedom, which remain truly three dimensional. Namely, the transverse spin appears due to the phaseshifted (e.g., imaginary) longitudinal $z$ component of the field, while the transverse momentum is also related to the 


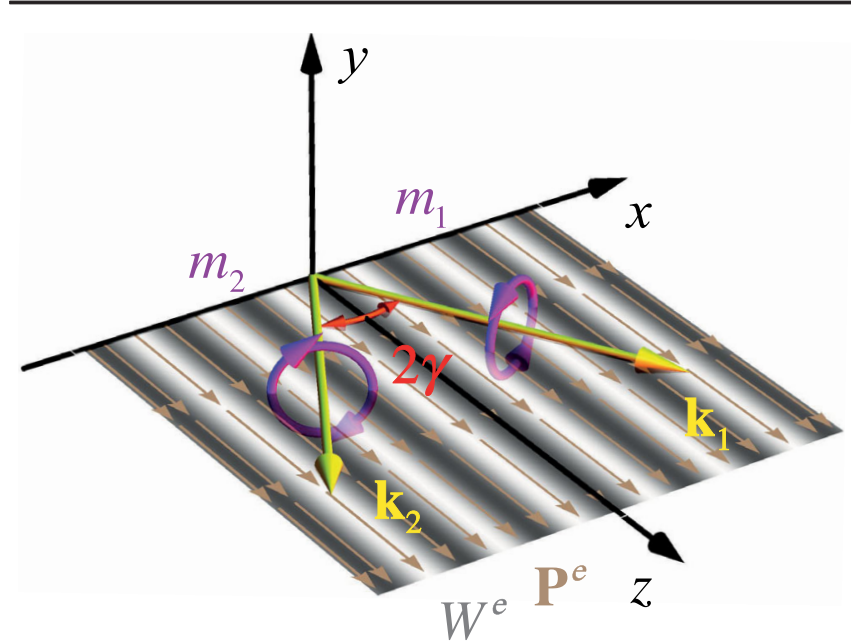

FIG. 1. Interference of two polarized plane waves. Two waves having equal amplitudes and wave vectors $\mathbf{k}_{1,2}$ with an angle $2 \gamma$ between them propagate and interfere in the $(x, z)$ plane. The wave polarizations are characterized by complex parameters $m_{1,2}$, and here the case of opposite circular polarizations $m_{1}=$ $-m_{2}=i$ (i.e., the Stokes parameters $\sigma_{1}=-\sigma_{2}=1$ ) is shown. Here and in Figs. 2 and 3, the gray scale plot represents the distribution of the electric energy density $W^{e}(x)$, Eqs. (4) and (9). The in-plane brown arrows show the electric part of the canonical momentum density of light $\mathbf{P}^{e}(x)$, Eqs. (5) and (10). This canonical momentum determines the energy transport and the optical pressure, and it is directed along the $z$ axis independently of the wave polarizations.

in-plane $x$ inhomogeneity of the field intensity. In the case of evanescent waves [10], the imaginary component of the single complex wave vector $\mathbf{k}=k_{z} \overline{\mathbf{z}}+i \kappa \overline{\mathbf{x}}$ provides for both of these features. In contrast, for propagating waves, considered here, at least two real wave vectors $\mathbf{k}_{1,2}=$ $k_{z} \overline{\mathbf{z}} \pm k_{x} \overline{\mathbf{x}}$ are needed to generate the truly threedimensional field and the in-plane $x$ inhomogeneity from the interference (see Fig. 1). Therefore, unlike the evanescent-wave case, where the extraordinary transverse quantities preserve constant signs in the $x>0$ half-space and only decay exponentially together with the field intensity, their counterparts in the two-wave field oscillate and change signs across the interference pattern.

To investigate the manifestations of the transverse momentum and spin AM in light-matter interactions, we calculate the optical forces and torques on a Mie particle immersed in the two-wave interference field. Remarkably, depending on the particle's position and wave polarizations, the particle can experience transverse torque about the out-of-plane axis, even in the case of linearly in-plane polarized incident waves with zero helicity. Furthermore, the particle can undergo a transverse force orthogonal to the wave vectors and strongly dependent on the wave polarization. These intriguing results, supported by both analytical theory and exact numerical simulations, call for experimental verification.

\section{MOMENTUM AND SPIN IN A TWO-WAVE INTERFERENCE FIELD}

Throughout this paper, we consider monochromatic electric and magnetic fields, $\mathcal{E}(\mathbf{r}, t)=\operatorname{Re}\left[\mathbf{E}(\mathbf{r}) e^{-i \omega t}\right]$ and $\mathcal{H}(\mathbf{r}, t)=\operatorname{Re}\left[\mathbf{H}(\mathbf{r}) e^{-i \omega t}\right]$, and use Gaussian units. All of the properties we discuss hold in free space, but to conform to optical-manipulation experiments using water or oil, we assume a homogeneous medium with real permittivity $\varepsilon$, permeability $\mu$, and refractive index $n=\sqrt{\varepsilon \mu}$.

The field we consider is a superposition of two plane waves with arbitrary polarizations propagating in the $(x, z)$ plane at an angle $2 \gamma$ between their wave vectors (see Fig. 1):

$$
\mathbf{k}_{1,2}=k(\cos \gamma \overline{\mathbf{z}} \pm \sin \gamma \overline{\mathbf{x}}) .
$$

Here, $k=n \omega / c$, the two signs correspond to the indices 1 and 2, and, hereafter, $\overline{\mathbf{x}}, \overline{\mathbf{y}}$, and $\overline{\mathbf{z}}$ denote the unit vectors of the corresponding axes. The complex electric fields of the two waves can be written as

$$
\mathbf{E}_{1,2}=\frac{A}{\sqrt{1+\left|m_{1,2}\right|^{2}}}\left(\cos \gamma \overline{\mathbf{x}}+m_{1,2} \overline{\mathbf{y}} \mp \sin \gamma \overline{\mathbf{z}}\right) e^{i \Phi_{1,2}}
$$

where $\Phi_{1,2}=k(z \cos \gamma \pm x \sin \gamma)$ are the wave phases and we assume that the two waves have equal real electric-field amplitudes $A$. In Eq. (2), $m_{1,2}$ are the complex parameters describing the wave polarizations [10,28]. The corresponding normalized Stokes parameters characterizing the degrees of the vertical (horizontal), diagonal $45^{\circ}\left(-45^{\circ}\right)$, and right-hand (left-hand) circular polarizations on the Poincaré sphere are, respectively,

$$
\begin{aligned}
\tau_{1,2} & =\frac{1-\left|m_{1,2}\right|^{2}}{1+\left|m_{1,2}\right|^{2}}, \\
\chi_{1,2} & =\frac{2 \operatorname{Re}\left(m_{1,2}\right)}{1+\left|m_{1,2}\right|^{2}}, \\
\sigma_{1,2} & =\frac{2 \operatorname{Im}\left(m_{1,2}\right)}{1+\left|m_{1,2}\right|^{2}} .
\end{aligned}
$$

Thus, $\sigma_{1,2}$ are the helicities of the two waves. The wave magnetic fields $\mathbf{H}_{1,2}$ corresponding to Eq. (2) are given in Supplemental Material [29], and the resulting interference fields are $\mathbf{E}=\mathbf{E}_{1}+\mathbf{E}_{2}$ and $\mathbf{H}=\mathbf{H}_{1}+\mathbf{H}_{2}$.

The main local dynamical characteristics of an optical field are the time-averaged densities of energy $W$, momentum $\mathbf{P}$, and spin AM S. They are described by the following equations with separate electric and magnetic contributions $[5,10,27,30]$ :

$$
W=W^{e}+W^{m}=\frac{g \omega}{2}\left(\varepsilon|\mathbf{E}|^{2}+\mu|\mathbf{H}|^{2}\right),
$$




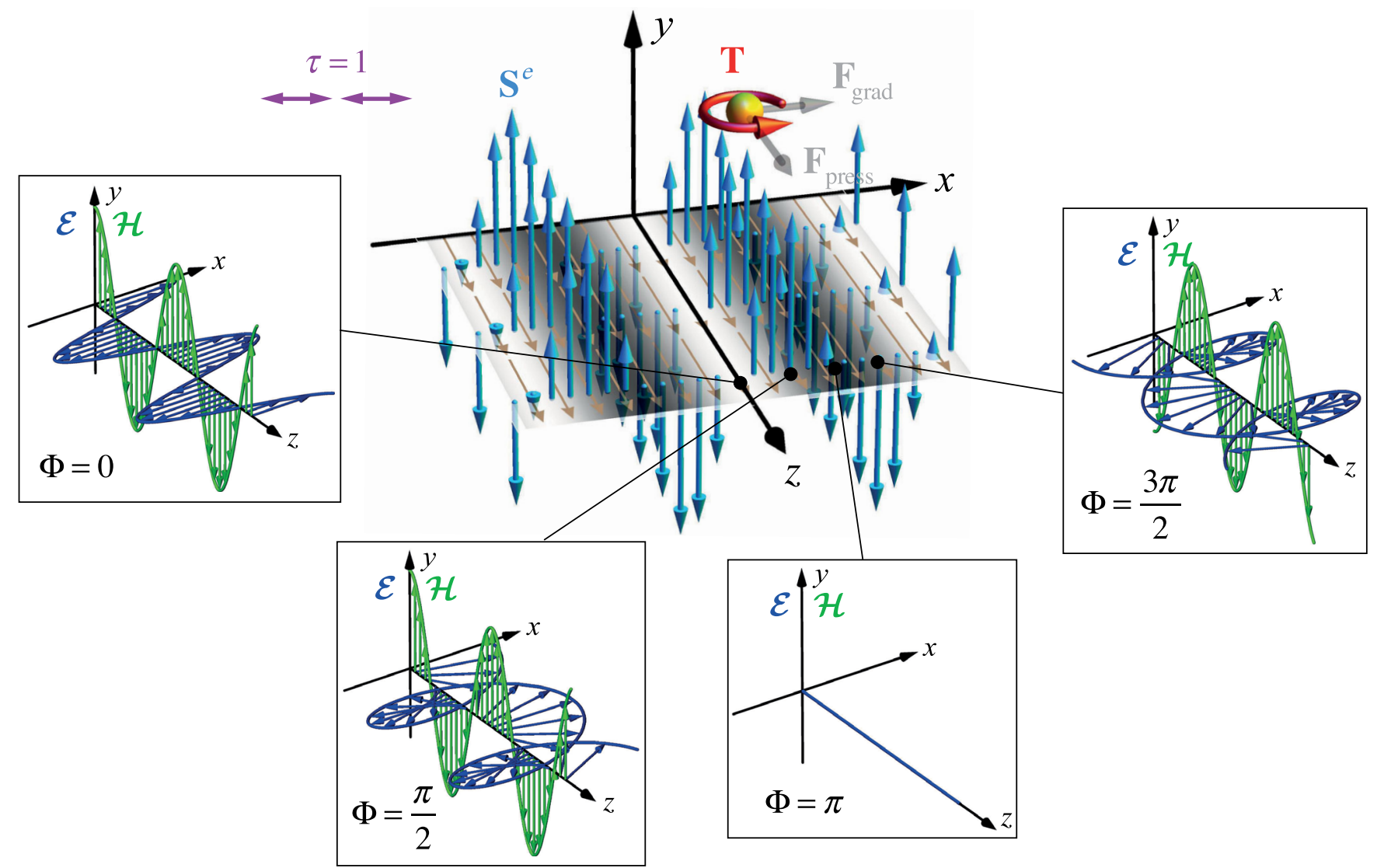

FIG. 2. Transverse helicity-independent spin in the two-wave interference field. Distribution of the electric spin AM density [Eq. (11)] $\mathbf{S}^{e}(x)$ is shown here for the simplest case of linearly in-plane polarized waves, $m_{1}=m_{2}=0$, i.e., the Stokes parameters $(\tau, \chi, \sigma)=$ $(1,0,0)$ (shown schematically in purple). Despite the seemingly $(x, z)$-planar character of the problem without any helicity, the transverse $y$-directed spin AM density appears. The inset panels display instantaneous electric and magnetic field distributions, $\mathcal{E}(\mathbf{r}, 0)$ and $\mathcal{H}(\mathbf{r}, 0)$, as functions of $z$ at different $x$ positions, indicated by the values of the phase $\Phi=2 k x \sin \gamma$. These distributions show that the transverse spin arises from the cycloidlike in-plane distribution of the electric field (cf. the evanescent-wave case [9-12]) with the direction of rotation dependent on $\Phi$. An absorbing probe particle is shown here at the position corresponding to $\Phi=\pi / 2$, and the optical forces and torques are indicated schematically. The transverse spin AM is locally transferred to the particle, thereby exerting a radiation torque [Eq. (15)] $\mathbf{T} \propto \mathbf{S}^{e}$ (shown in red) (see Fig. 4 for numerical simulations). The particle also experiences in-plane radiationpressure and gradient forces [Eq. (14)] [shown in gray].

$$
\begin{gathered}
\mathbf{P}=\mathbf{P}^{e}+\mathbf{P}^{m}=\frac{g}{2} \operatorname{Im}\left[\frac{1}{\mu} \mathbf{E}^{*} \cdot(\nabla) \mathbf{E}+\frac{1}{\varepsilon} \mathbf{H}^{*} \cdot(\nabla) \mathbf{H}\right], \\
\mathbf{S}=\mathbf{S}^{e}+\mathbf{S}^{m}=\frac{g}{2} \operatorname{Im}\left(\frac{1}{\mu} \mathbf{E}^{*} \times \mathbf{E}+\frac{1}{\varepsilon} \mathbf{H}^{*} \times \mathbf{H}\right),
\end{gathered}
$$

where $g=(8 \pi \omega)^{-1}$ in Gaussian units. It should be emphasized that Eq. (5) determines the so-called canonical (or orbital) momentum of light, which can be associated with the local phase gradient of the field [5] and which follows from the Noether theorem and canonical energy-momentum tensor [30]. This momentum is responsible for the energy transfer, the radiation pressure, and also appears in quantum weak measurements of the photon momentum (see Refs. [5,7-10,27,30-32]). Thus, it is this canonical momentum, but not the Poynting vector, that represents the directly observable momentum of light. In particular, the momentum (5) is responsible for the "supermomentum" effects
$|\mathbf{P}|>W / c$ [4-8], which are impossible with the Poynting vector that never exceeds $W / c$ in absolute value [9].

Nonetheless, below we also use the complex Poynting momentum I [2], which plays a role in higher-order lightmatter interactions [10] (or interactions with complex particles [27]):

$$
\boldsymbol{\Pi}=\frac{g k}{n}\left(\mathbf{E}^{*} \times \mathbf{H}\right) .
$$

The real part of the Poynting vector (7) differs from the canonical momentum (5) by the so-called spin momentum $\mathcal{P}_{S}$, which was introduced in 1939 by Belinfante to explain the spin AM of quantum particles within field theory [33-36] (see also Refs. [5,9,10,30,31]):

$$
\begin{aligned}
\operatorname{Re}(\boldsymbol{\Pi}) & =\mathbf{P}+\mathcal{P}_{S}, \\
\mathcal{P}_{S} & =\frac{1}{2} \nabla \times \mathbf{S} .
\end{aligned}
$$



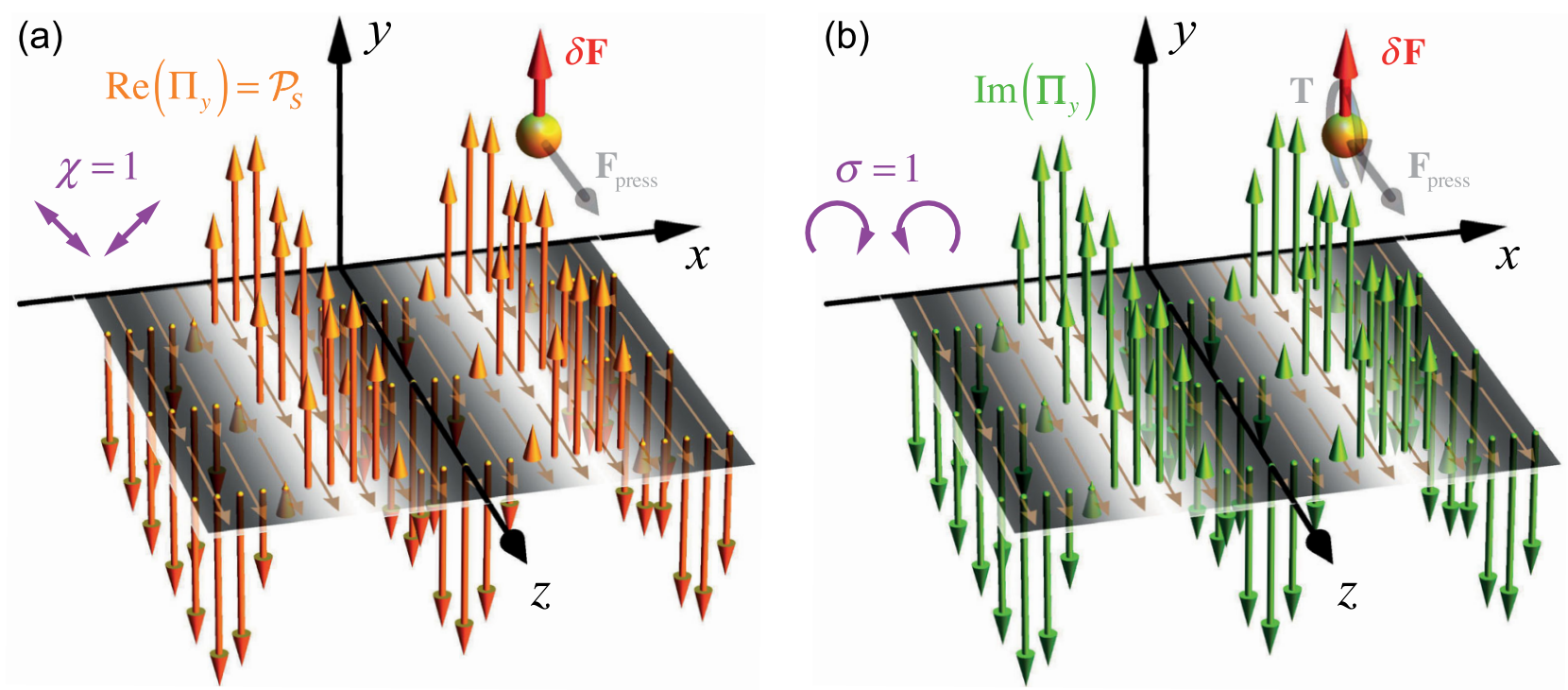

FIG. 3. Transverse polarization-dependent momenta in the two-wave interference field. (a) Distribution of Belinfante's spin momentum density [Eq. (12)] $\mathcal{P}_{S}(x)$ ["virtual" part of the real Poynting vector $\operatorname{Re}(\boldsymbol{\Pi})$ ] in the case of diagonally polarized waves with $m_{1}=-m_{2}=1$, i.e., the Stokes parameters $(\tau, \chi, \sigma)=(0,1,0)$ (cf. the Fedorov-Imbert transverse momentum in evanescent waves [10]). (b) Distribution of the $y$ component of the imaginary Poynting momentum density $\operatorname{Im}\left[\Pi_{y}(x)\right]$ [Eq. (13)] for circularly polarized waves with $m_{1}=-m_{2}=i$, i.e., the Stokes parameters $(\tau, \chi, \sigma)=(0,0,1)$. Despite the planar two-wave interference, both of these noncanonical momenta have the transverse $y$-directed components, which are strongly polarization dependent. Namely, the distributions in (a) and (b) are flipped when the polarizations are changed to the opposite: $\chi=-1$ and $\sigma=-1$. An absorbing probe particle is shown here at the $x$ position corresponding to $\Phi=\pi$, and the optical forces and torques are indicated schematically. The spin and imaginary Poynting momenta do not exert radiation pressure in the dipole-coupling approximation, but do cause a weak polarization-dependent optical force [Eq. (16)] (shown in red), $\delta F_{y} \propto \operatorname{Re}\left(\Pi_{y}\right)=\mathcal{P}_{S y}$ and $\delta F_{y} \propto \operatorname{Im}\left(\Pi_{y}\right)$ in the higher-order approximation (see Fig. 4 for numerical simulations). The particle also experiences the action of the in-plane radiation-pressure force [Eq. (14)] and torque [Eq. (15)] (shown in gray); the latter corresponds to the helicity-dependent $x$-directed spin [Eq. (11)] of the interfering waves with opposite circular polarizations [37].

Importantly, the divergenceless spin momentum $\mathcal{P}_{S}$ does not transfer energy, does not exert optical pressure on spherical dipole particles, and is often considered as "virtual," i.e., nonobservable. It is this momentum that appears as the enigmatic Fedorov-Imbert momentum in evanescent waves $[10,14,15]$, which is orthogonal to the wave vector and depends on the polarization helicity.

Substituting electric and magnetic fields of the superposition of two waves (2) into the general equations (4)-(6), we calculate the energy, momentum, and spin AM distributions in the two-wave interference. Formulas for the generic polarizations $m_{1}$ and $m_{2}$ are given in Supplemental Material [29], while here, for the sake of simplicity, we consider a particular case of the "mirrorsymmetric" polarizations, $m_{1}=-m_{2} \equiv m$, with the Stokes parameters $\left(\tau_{1}, \chi_{1}, \sigma_{1}\right)=\left(\tau_{2},-\chi_{2},-\sigma_{2}\right) \equiv(\tau, \chi, \sigma)$. Omitting the common factor $\varepsilon g A^{2}$ in all dynamical characteristics, this yields

$$
\begin{gathered}
W^{e, m}=\omega\left[1+\left(\tau \cos ^{2} \gamma \mp \sin ^{2} \gamma\right) \cos \Phi\right], \\
\mathbf{P}^{e, m}=\frac{\cos \gamma}{c n} W^{e, m} \overline{\mathbf{z}}
\end{gathered}
$$

$$
\begin{aligned}
\mathbf{S}^{e, m}= & \frac{1}{n^{2}}\left[\sigma \sin \gamma(1 \mp \cos \Phi) \overline{\mathbf{x}}+\frac{\tau \pm 1}{2} \sin 2 \gamma \sin \Phi \overline{\mathbf{y}}\right. \\
& +\chi \cos \gamma \sin \Phi \overline{\mathbf{z}}]
\end{aligned}
$$

where the two signs correspond to the indices $e$ and $m$, while $\Phi=\Phi_{1}-\Phi_{2}=2 k x \sin \gamma$ is the phase difference that determines the interference pattern (see Fig. 1). In a similar way, we also find the spin momentum and imaginary Poynting vector, Eqs. (7) and (8):

$$
\begin{gathered}
\mathcal{P}_{S}=\frac{k \sin 2 \gamma}{n^{2}}[-\chi \cos \Phi \overline{\mathbf{y}}+\tau \sin \gamma \cos \Phi \overline{\mathbf{z}}], \\
\operatorname{Im}(\boldsymbol{\Pi})=-\frac{2 k \sin \gamma}{n^{2}}[\sin \Phi \overline{\mathbf{x}}+\sigma \cos \gamma \cos \Phi \overline{\mathbf{y}}] .
\end{gathered}
$$

Equations (9)-(13) are the key equations of this work. The energy densities and canonical momenta, Eqs. (9) and (10), correspond to the picture of the two-wave interference, which is intuitively clear and has been known for decades. Namely, $W^{e, m}(x)$ contains the usual interference fringes determined by $\cos \Phi=\cos (2 k x \sin \gamma)$, while $\mathbf{P}^{e, m}(x)$ is naturally directed along the $z$ axis and corresponds to the group propagation velocity $v_{g}=P_{z}^{e, m} c^{2} / W^{e, m}=c \cos \gamma / n$ [7], 
Fig. 1. In contrast, Eqs. (11)-(13) reveal unexpected and counterintuitive dynamical features in such a primitive system. Despite the seemingly planar $(x, z)$ geometry of the problem, the spin AM and the complex Poynting momentum have transverse out-of-plane $y$ components.

The second term in Eq. (11) describes the transverse $y$-directed spin AM, which is independent of the helicity $\sigma$ and can appear even for linear in-plane polarization, Fig. 2. Similar transverse spin was previously described only in evanescent fields [9-13]. This transverse spin density varies sinusoidally across the interference fringes, so that the integral (i.e., $\Phi$-averaged) spin $\mathrm{AM}$ vanishes at $\sigma=0$. The instantaneous $t=0$ distribution of the electric and magnetic fields, $\mathcal{E}(\mathbf{r}, t)$ and $\mathcal{H}(\mathbf{r}, t)$, shown in Fig. 2, illuminates the origin of this transverse spin $S_{y}^{e}$. Interference of the $x$ and $z$ components of the electric wave fields (2), which arrive at the observation point with different phases, results in the cycloidal field distributions and in-plane rotation upon the propagation along the $z$ axis, cf. Refs. [9-13] (see also Supplemental Material [29]).

Note that the integral spin AM in the chosen polarization configuration $m_{1}=-m_{2}$ originates from the first term in Eq. (11): $\langle\mathbf{S}\rangle=2 \sigma n^{-2} \sin \gamma \overline{\mathbf{x}}$. It is proportional to the helicity $\sigma$, as expected, but it is directed along the $x$ axis, i.e., also orthogonally to the main propagation direction. This spin AM is similar to that recently described in Ref. [37], and it can be explained by the summation of the usual spin AM from the two waves: $\left(\sigma_{1} \mathbf{k}_{1}+\sigma_{2} \mathbf{k}_{2}\right) / k=2 \sigma \sin \gamma \overline{\mathbf{x}}$.

Next, the first term in Eq. (12) and the second one in Eq. (13) describe the real and imaginary parts of the transverse $y$ directed complex Poynting momentum [Eq. (7)]: $\operatorname{Re}\left(\Pi_{y}\right)=\mathcal{P}_{S y}$ and $\operatorname{Im}\left(\Pi_{y}\right)$, Fig. 3. In contrast to the longitudinal canonical momentum (10), both of these transverse parts are strongly polarization dependent $(\chi$ and $\sigma$ dependent for the chosen configuration $m_{1}=-m_{2}$; see Supplemental Material [29] for other cases). Importantly, the real part of the transverse Poynting vector is a pure Belinfante's spin momentum [Eq. (8)], which is analogous to the helicity-dependent transverse momentum in evanescent waves (first found by Fedorov and Imbert [14,15] and explained only very recently [10]). It emerges because of the spatial $x$ inhomogeneity of the usual longitudinal $z$-directed spin $S_{z}$, Eq. (11), which is in turn produced by the field rotation in the $(x, y)$ plane, Eq. (6). Both parts of $\Pi_{y}$ do not transport energy, do not exert the usual optical pressure, and have sinusoidal distributions across the interference fringes, Fig. 3. Nonetheless, below we show that they do reveal themselves in light-matter interactions, and, hence, can be detected experimentally.

\section{MECHANICAL ACTION ON PROBE PARTICLES}

We now describe manifestations of the unusual dynamical characteristics [Eqs. (9)-(13)] of the two-wave field in light-matter interactions. For this purpose, we consider the field interaction with a small spherical probe particle. This approach is verified in numerous experimental [18,20-22] and theoretical [10,23-27] studies. The particle is absorptive and is characterized by subwavelength radius $r$ and complex electric and magnetic polarizabilities $\alpha^{e, m}$.

For typical nonmagnetic materials, the electric polarizability is much higher than the magnetic one, because $\alpha^{e} \propto(k r)^{3}$ and $\alpha^{m} \propto(k r)^{5}$ for $k r \ll 1$ (see the Appendix and Supplemental Material [29]). Therefore, for Rayleigh particles, the leading-order light-matter interaction is the electric-dipole coupling. It results in the following optical force $\mathbf{F}$ and torque $\mathbf{T}$ on the particle [10,23-27]:

$\mathbf{F}=g^{-1}\left[\frac{1}{2 \omega \varepsilon} \operatorname{Re}\left(\alpha^{e}\right) \nabla W^{e}+\mu \operatorname{Im}\left(\alpha^{e}\right) \mathbf{P}^{e}\right] \equiv \mathbf{F}_{\text {grad }}+\mathbf{F}_{\text {press }}$,

$$
\mathbf{T}=g^{-1} \operatorname{Im}\left(\alpha^{e}\right) \mathbf{S}^{e},
$$

where the force consists of the gradient and radiationpressure contributions. The radiation-pressure force in Eq. (14) and torque [Eq. (15)], both proportional to $\operatorname{Im}\left(\alpha^{e}\right)$, characterize the rate of the momentum and AM transfer from light to the particle via the photon-absorption mechanism.

Being proportional to the electric parts of the canonical momentum density $\mathbf{P}^{e}$ [Eq. (5)] and spin AM density $\mathbf{S}^{e}$ [Eq. (6)], the radiation-pressure force and torque on the particle naturally measure these local characteristics of the field $[7,10,21,22,27]$, including the transverse spin $S_{y}^{e}(x)$. In addition to the asymptotic analytical results [Eq. (15)], Fig. 4(a) shows exact numerical calculations of the torque on a gold Mie particle, with $0<k r<4$, suspended in water (see the Appendix) and interacting with the two-wave interference field with linear polarizations $m=0$ and $m=$ $\infty$ (Stokes parameter $\tau= \pm 1$ ) at the $x$ position corresponding to $\Phi=\pi / 2$. One can clearly see the transverse torque $T_{y}$ about the $y$ axis revealing the transverse spin AM density $S_{y}^{e}(x)$ [Eq. (11)], see Fig. 2, which will change the sign for the $x$ position, corresponding to $\Phi=3 \pi / 2$. The particle simultaneously experiences the action of the inplane radiation-pressure and gradient forces in this location, but these could be balanced in experiments trapping the particle at a desired $(x, z)$ point.

Remarkably, in the above dipole-coupling approximation, the transverse components of the complex Poynting momentum, Eqs. (12) and (13), have no effect on the particle. However, they do appear in the higher-order interaction involving cross electric-magnetic dipole-dipole terms proportional to $\alpha^{e} \alpha^{m *} \propto(k r)^{8}$ [10]. Such weak interaction is negligible for Rayleigh particles, but it becomes noticeable for larger Mie particles with $k r \sim 1$. This results in the weak force correction $[10,24,25]$ : 


\section{$\mathbf{T} / T_{0}$}
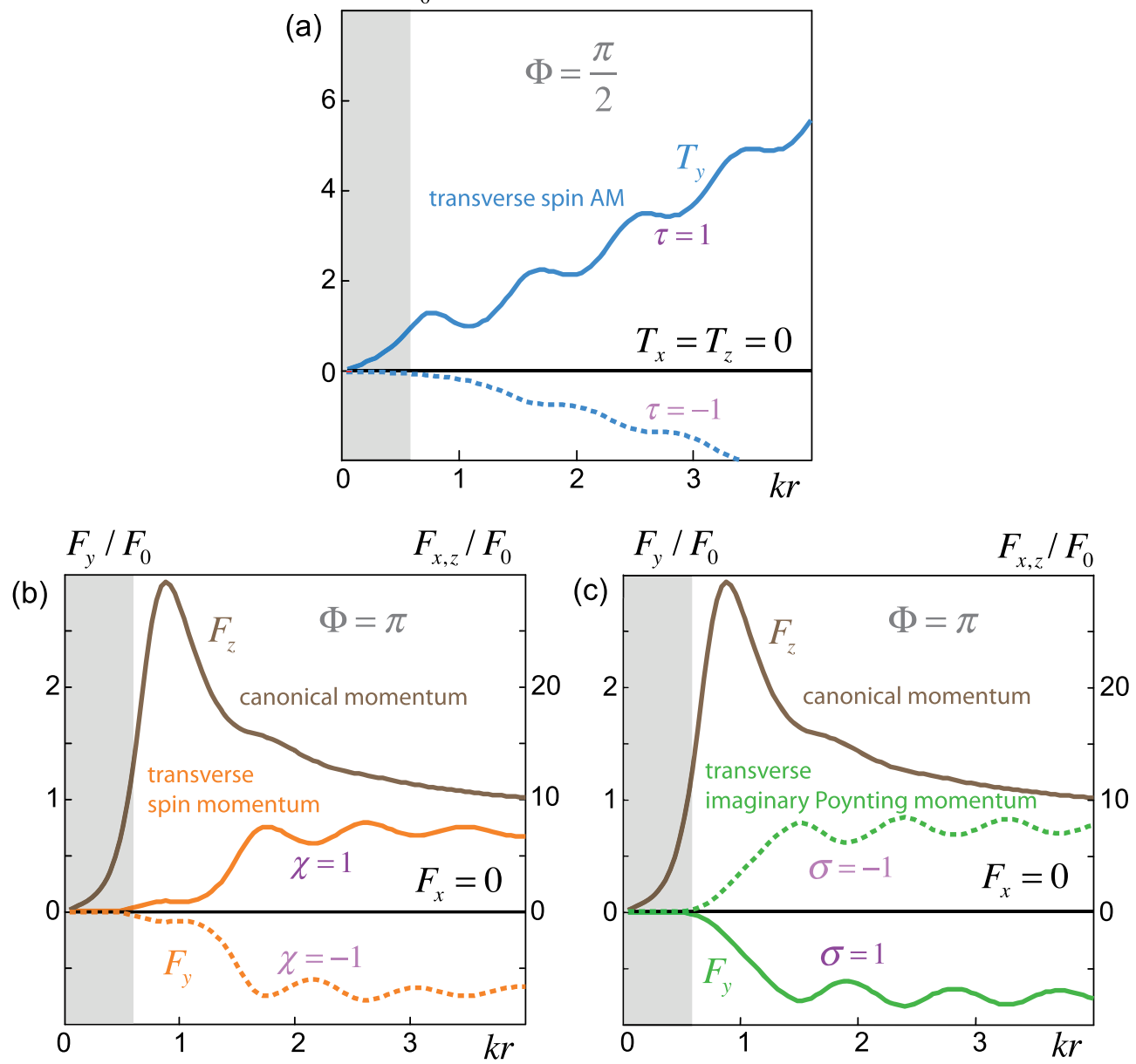

FIG. 4. Optical forces and torques on a gold Mie particle in the two-wave interference field. Exact numerical calculations of the forces and torques exerted by the two-wave interference field [Eq. (2)] with $\gamma=0.1$ and different polarizations on a gold Mie particle suspended in water (see the Appendix). The forces and torques (normalized by the factors $F_{0}=g r^{2} A^{2}$ and $T_{0}=F_{0} / k$ ) are plotted as functions of the dimensionless radius of the particle, $k r$. The light gray areas schematically indicate the Rayleigh dipole-approximation range $k r \ll 1$. (a) Optical torques for the simplest case of linear polarizations $m=0$ and $m=\infty$, i.e., the Stokes parameters $\tau= \pm 1$, and particle's $x$ position corresponding to $\Phi=\pi / 2$. The strong transverse torque clearly indicates the presence of a transverse helicityindependent spin AM: $T_{y} \propto S_{y}^{e}=S_{y}$ for the in-plane polarization $\tau=1$; see Eqs. (11) and (15) and Fig. 2. The same torque becomes weak and negative for the $\tau=-1$ polarization, because in this case the transverse spin has magnetic origin, $S_{y}=S_{y}^{m}$, and it is weakly coupled to the nonmagnetic gold particle. (b),(c) Optical forces for the diagonal and circular wave polarizations $m= \pm 1$ and $m= \pm i$, i.e., the Stokes parameters $\chi= \pm 1$ and $\sigma= \pm 1$, and particle's $x$ position corresponding to $\Phi=\pi$. The strongest force is the longitudinal radiation-pressure force proportional to the $z$-directed canonical momentum of light, $F_{z} \propto P_{z}^{e}$, Eqs. (10) and (14). The gradient force is absent because the particle is located in the maximum of the electric-field intensity: $F_{x} \propto \nabla_{x} W^{e}=0$. Importantly, a weak polarizationdependent transverse force $F_{y}$ appears beyond the Rayleigh dipole approximation, i.e., at $k r \sim 1$. This force [Eq. (16)] indicates the presence of the transverse $\chi$-dependent Belinfante spin momentum $\delta F_{y} \propto \operatorname{Re}\left(\Pi_{y}\right)=P_{S y}$ and $\sigma$-dependent imaginary Poynting momentum $\delta F_{y} \propto \operatorname{Im}\left(\Pi_{y}\right)$, Eqs. (12) and (13) and Fig. 3.

$\delta \mathbf{F}=g^{-1} \frac{k^{3}}{3}\left[-\operatorname{Re}\left(\alpha^{e} \alpha^{m *}\right) \operatorname{Re}(\boldsymbol{\Pi})+\operatorname{Im}\left(\alpha^{e} \alpha^{m *}\right) \operatorname{Im}(\boldsymbol{\Pi})\right]$.

The in-plane $(x, z)$ components of this force are negligible compared to the radiation-pressure and gradient forces (14), but the transverse component $\delta F_{y}$ is the only force in the $y$ direction. It has two contributions, proportional to the transverse Belinfante's spin momentum (12),
$\operatorname{Re}\left(\Pi_{y}\right)=\mathcal{P}_{S y}$, and to the imaginary Poynting momentum $\operatorname{Im}\left(\Pi_{y}\right)$ [Eq. (13)]. Therefore, both of these transverseforce components are strongly polarization dependent, and are proportional to the Stokes parameters $\chi$ and $\sigma$, respectively.

Figures 4(b) and 4(c) depict the results of the exact numerical calculations of the forces exerted on the same golden Mie particle (see the Appendix) in the two-wave interference fields with polarizations $m= \pm 1$ and $m= \pm i$ 
(Stokes parameters $\chi= \pm 1$ and $\sigma= \pm 1$ ) at the $x$ position, corresponding to $\Phi=\pi$. Alongside the strong polarizationindependent radiation-pressure force $F_{z}$, one can see extraordinary transverse forces $F_{y}$ changing their signs upon the flip of the Stokes parameters $\chi$ and $\sigma$ (these forces also have the opposite signs at the $x$ position, corresponding to $\Phi=0$ ). Importantly, for Mie particles with $k r \sim 1$, the weak force $F_{y}$ is only 1 order of magnitude below the usual radiation-pressure force $F_{z}$, and, therefore, it is clearly detectable in standard optical-manipulation experiments. This proves the observability of the transverse spin momentum [Eq. (12)] and imaginary Poynting momentum [Eq. (13)], Fig. 3.

It is worth remarking that the directions of the forces depend on the parameters of the particle, and Figs. 4(b) and 4(c) show the transverse forces $F_{y}$ corresponding to the negative factors $\operatorname{Re}\left(\alpha^{e} \alpha^{m *}\right)<0$ and $\operatorname{Im}\left(\alpha^{e} \alpha^{m *}\right)<0$ for the chosen gold particle (see the Appendix). Note also that there is no gradient force at $\Phi=0, \pi$, which offers a natural trapping of the particle in the $x$ positions with maximum transverse forces. (The stable or unstable character of these positions depends on the sign of the gradient forces in their vicinity, which in turn is determined by the parameters of the particle [18].)

Strikingly, the field characteristics [Eqs. (9)-(13)], dipole interactions [Eqs. (14) and (15)], and the weak force correction [Eq. (16)] are not merely leading-order terms in a series of multiple light-matter interaction orders. Being asymptotic with respect to the particle size $k r$ at $k r \ll 1$, Eqs. (9)-(16) precisely keep the dependencies of optical forces and torques on wave polarizations $(\tau, \chi, \sigma)$ and phases $\Phi$ even for larger Mie particles with $k r>1$. This can be seen in detailed numerical analysis given in the Supplemental Material [29]. Thus, the above description is indeed fundamental and complete.

We also note from Eqs. (11)-(13) that all of the transverse spin and momentum phenomena discussed here depend on the angle $\gamma$ as $\propto \sin 2 \gamma$, which enters as an overall scaling factor for these phenomena. For the numerical simulations, we chose the reasonably small angle $\gamma=0.1$ (paraxial propagation), which provides the period of the fringes, $\pi /(k \sin \gamma)$, of about five wavelengths. This is sufficient for placing a wavelength-order probe in the required $x$ position between the fringes.

\section{CONCLUSIONS}

To summarize, we show that one of the simplest optical systems - two interfering plane waves-still provides surprises, exhibiting rather rich and unexpected local dynamical properties. Despite the seemingly planar character of the system (which can also reveal remarkable fine features in wave reflection or refraction [38]), we find that the twowave field carries a nonzero transverse (out-of-plane) spin and momentum densities. These quantities appear because the planar wave vectors determine only the extrinsic degrees of freedom of the wave field, while the polarization degrees of freedom and their associated properties remain truly three dimensional.

Remarkably, the transverse spin AM is independent of the wave helicity and appears even for linearly polarized waves. On the contrary, the transverse component of the complex Poynting momentum is strongly polarization dependent, the real part of this transverse momentum being Belinfante's spin momentum. The transverse Poynting momentum does not exert radiation pressure in dipolecoupling interactions, but it appears in the higher-order interactions with larger Mie particles and can be detected. We perform exact numerical calculations of the forces and torques exerted on a particle in the interference field and prove the straightforward observability of the above extraordinary dynamical features.

The fact that the transverse spin and momentum can be obtained from simple planar propagating fields is very important for experiments, as the latter are much easier to generate and design than evanescent fields considered in previous works [9-15]. Our findings offer a new vision for the fundamental properties of propagating optical fields and pave the way for nontrivial optical manipulations of small particles.

Note added in proof.-After this work was accepted, we became aware of two experimental papers $[39,40]$, which confirm our theoretical findings. First, a weak helicitydependent transverse force was detected in [39] for a Mie particle in interference of the orthogonal linear- and circularly-polarized plane waves. Although this force was interpreted there using "Aharonov-Bohm effect" arguments, it can be clearly explained as the force (16) proportional to the transverse imaginary part of the Poynting vector. The second term in Eq. (S10) in the Supplemental Material [29], with $2 \gamma=\pi / 2, m_{1}=i \sigma$, and $m_{2}=0$, describes this transverse Poynting-vector component. Second, the transverse helicityindependent spin AM density was measured in [40] in a focused radially-polarized Gaussian beam. Since the beam is produced by interference of multiple plane waves, our twowave system can be considered as a toy model for the interference phenomena in the beam [38].

\section{ACKNOWLEDGMENTS}

This work was partially supported by the RIKEN Interdisciplinary Theoretical Science Research Group (iTHES) Project, Multi-University Research Initiative (MURI) Center for Dynamic Magneto-Optics, Japan Society for the Promotion of Science (JSPS)-Russian Foundation for Basic Research (RFBR) Contract No. 1202-92100, and a Grant-in-Aid for Scientific Research (S).

\section{APPENDIX: CALCULATIONS OF OPTICAL FORCES AND TORQUES ON MIE PARTICLES}

For numerical simulations, we consider the two-wave interference field [Eq. (2)] with the vacuum wavelength 
$\lambda_{0}=2 \pi c / \omega=650 \mu \mathrm{m}$ and angle $\gamma=0.1$ interacting with a spherical gold particle of radius $r$ suspended in water. Accordingly, the medium (water) is characterized by $\varepsilon=$ 1.77 and $\mu=1$, whereas the particle electric and magnetic constants are $\varepsilon_{p}=-12.2+3.0 i$ and $\mu_{p}=1$.

To estimate the magnitudes of the optical forces and torques in the small-particle approximation $k r \ll 1$, Eqs. (14)-(16), one can use Eq. (S26) of the Supplemental Material [29], which describes the effective electric and magnetic polarizabilities of a nonmagnetic particle in the leading order in $k r$. With the above parameters, this yields

$$
\begin{aligned}
\alpha^{e} & \simeq\left(1.28 \times 10^{-3}+1.56 \times 10^{-4} i\right)(k r)^{3} \mu \mathrm{m}^{3}, \\
\alpha^{m} & \simeq\left(-1.24 \times 10^{-4}+2.66 \times 10^{-5} i\right)(k r)^{5} \mu \mathrm{m}^{3}, \\
\alpha^{e} \alpha^{m *} & \simeq\left(-1.55 \times 10^{-7}-5.37 \times 10^{-8} i\right)(k r)^{8} \mu \mathrm{m}^{6} .
\end{aligned}
$$

For exact calculations with a particle of arbitrary radius $r$, we use the standard Mie theory [41], generalized for the case of two incident plane waves. Namely, using the Mie solution for a single plane wave, we determine the scattered electromagnetic fields $\left(\mathbf{E}_{1}^{s}, \mathbf{H}_{1}^{s}\right)$ and $\left(\mathbf{E}_{2}^{s}, \mathbf{H}_{2}^{s}\right)$ for each of the incident waves, $\left(\mathbf{E}_{1}, \mathbf{H}_{1}\right)$ and $\left(\mathbf{E}_{2}, \mathbf{H}_{2}\right)$. Hence, the total field, perturbed by the interaction with the particle, is

$$
\begin{aligned}
\mathbf{E}^{\mathrm{tot}} & =\mathbf{E}_{1}+\mathbf{E}_{2}+\mathbf{E}_{1}^{s}+\mathbf{E}_{2}^{s}, \\
\mathbf{H}^{\mathrm{tot}} & =\mathbf{H}_{1}+\mathbf{H}_{2}+\mathbf{H}_{1}^{s}+\mathbf{H}_{2}^{s} .
\end{aligned}
$$

Once the total field is known, its mechanical action on the particle is calculated via the standard procedures using the Maxwell stress tensor [2] $\hat{Q}=\left\{Q_{i j}\right\}, i, j=x, y, z$,

$$
\begin{aligned}
Q_{i j}= & g \omega \operatorname{Re}\left[\varepsilon E_{i}^{\mathrm{tot} *} E_{j}^{\mathrm{tot}}+\mu H_{i}^{\mathrm{tot} *} H_{j}^{\mathrm{tot}}\right. \\
& \left.-\frac{1}{2} \delta_{i j}\left(\varepsilon\left|\mathbf{E}^{\mathrm{tot}}\right|^{2}+\mu\left|\mathbf{H}^{\mathrm{tot}}\right|^{2}\right)\right],
\end{aligned}
$$

and the corresponding AM flux tensor $\hat{M}=\left\{M_{i j}\right\}$, $M_{i j}=e_{j k l} x_{k} Q_{l i}$. Here, $\delta_{i j}$ is the Kronecker delta, $e_{j k l}$ is the Levi-Civita symbol, and $\left\{x_{i}\right\}=\{x, y, z\}$. Integrating the stress tensor and the AM flux tensor components over any surface $\Sigma$ enclosing the particle (e.g., a sphere with radius $R>r$ ), we obtain the optical force and torque exerted on the particle:

$$
\begin{aligned}
& \mathbf{F}=\oint_{\Sigma} \hat{Q} \mathbf{n} d \Sigma=R^{2} \int_{\Omega} \hat{Q} \mathbf{n} d \Omega, \\
& \mathbf{T}=\oint_{\Sigma} \hat{M} \mathbf{n} d \Sigma=R^{2} \int_{\Omega} \hat{M} \mathbf{n} d \Omega .
\end{aligned}
$$

Here, $d \Omega=\sin \theta d \theta d \phi$ is the elementary solid angle and $\mathbf{n}=(\sin \theta \cos \phi, \sin \theta \sin \phi, \cos \theta)^{T}$ is the unit vector of the outer normal to the surface of the sphere. Finally, the forces and torques calculated using the above method are normalized by the factors $F_{0}=g r^{2} A^{2}$ and $T_{0}=F_{0} / k$, and are plotted in Fig. 4 as well as in Figs. S2 and S3 in the Supplemental Material [29]. Since $\alpha^{e} \propto(k r)^{3}$ in the Rayleigh limit $k r \ll 1$, such normalization implies linear growth with $k r$, at $k r \ll 1$, for the electric-dipole quantities [Eqs. (14) and (15)].

[1] R. Loudon and C. Baxter, Contribution of John Henry Poynting to the Understanding of Radiation Pressure, Proc. R. Soc. A 468, 1825 (2012).

[2] J. D. Jackson, Classical Electrodynamics, 3rd ed. (Wiley, New York, 1999).

[3] L. Allen, S. M. Barnett, and M. J. Padgett, Optical Angular Momentum (Institute of Physics Publishing, Bristol, 2003).

[4] S. Huard and C. Imbert, Measurement of Exchanged Momentum during Interaction between Surface-Wave and Moving Atom, Opt. Commun. 24, 185 (1978).

[5] M. V. Berry, Optical Currents, J. Opt. A 11, 094001 (2009).

[6] M. R. Dennis, A. C. Hamilton, and J. Courtial, Superoscillations in Speckle Patterns, Opt. Lett. 33, 2976 (2008).

[7] K. Y. Bliokh, A. Y. Bekshaev, A. G. Kofman, and F. Nori, Photon Trajectories, Anomalous Velocities, and Weak Measurements: A Classical Interpretation, New J. Phys. 15, 073022 (2013).

[8] S. M. Barnett and M. V. Berry, Superweak Momentum Transfer near Optical Vortices, J. Opt. 15, 125701 (2013).

[9] K. Y. Bliokh and F. Nori, Transverse Spin of a Surface Polariton, Phys. Rev. A 85, 061801(R) (2012).

[10] K. Y. Bliokh, A. Y. Bekshaev, and F. Nori, Extraordinary Momentum and Spin in Evanescent Waves, Nat. Commun. 5, 3300 (2014).

[11] A. Canaguier-Durand and C. Genet, Transverse Spinning of a Sphere in Plasmonic Field, Phys. Rev. A 89, 033841 (2014).

[12] C. Junge, D. O'Shea, J. Volz, and A. Rauschenbeutel, Strong Coupling between Single Atoms and Nontransversal Photons, Phys. Rev. Lett. 110, 213604 (2013); M. Neugebauer, T. Bauer, P. Banzer, and G. Leuchs, Polarization Tailored Light Driven Directional Optical Nanobeacon, Nano Lett. 14, 2546 (2014).

[13] J. Petersen, J. Volz, and A. Rauschenbeutel, Chiral Nanophotonic Waveguide Interface Based on Spin-Orbit Coupling of Light, Science 346, 67 (2014); D. O'Connor, P. Ginzburg, F. J. Rodriguez-Fortuno, G. A. Wurtz, and A. V. Zayats, Spin-orbit Coupling in Surface Plasmon Scattering by Nanostructures, Nat. Commun. 5, 5327 (2014).

[14] F. I. Fedorov, To the Theory of Total Reflection, Dokl. Akad. Nauk SSSR 105, 465 (1955); J. Opt. 15, 014002 (2013).

[15] C. Imbert, Calculation and Experimental Proof of the Transverse Shift Induced by Total Internal Reflection of a Circularly Polarized Light Beam, Phys. Rev. D 5, 787 (1972).

[16] C. Cohen-Tannoudji, J. Dupont-Roc, and G. Grynberg, Atom-Photon Interactions (Wiley-VCH, New York, 2004).

[17] S. Stenholm, The Semiclassical Theory of Laser Cooling, Rev. Mod. Phys. 58, 699 (1986). 
[18] D. G. Grier, A Revolution in Optical Manipulation, Nature (London) 424, 810 (2003).

[19] M. Aspelmeyer, T. J. Kippenberg, and F. Marquardt, Cavity Optomechanics, Rev. Mod. Phys. 86, 1391 (2014).

[20] M. E. J. Friese, T. A. Nieminen, N. R. Heckenberg, and H. Rubinsztein-Dunlop, Optical Alignment and Spinning of Laser-Trapped Microscopic Particles, Nature (London) 394, 348 (1998).

[21] A. T. O'Neil, I. MacVicar, L. Allen, and M. J. Padgett, Intrinsic and Extrinsic Nature of the Orbital Angular Momentum of a Light Beam, Phys. Rev. Lett. 88, 053601 (2002).

[22] V. Garcés-Chavéz, D. McGloin, M. J. Padgett, W. Dultz, H. Schmitzer, and K. Dholakia, Observation of the Transfer of the Local Angular Momentum Density of a Multiringed Light Beam to an Optically Trapped Particle, Phys. Rev. Lett. 91, 093602 (2003).

[23] A. Ashkin and J. P. Gordon, Stability of Radiation-Pressure Particle Traps: An Optical Earnshaw Theorem, Opt. Lett. 8, 511 (1983).

[24] M. Nieto-Vesperinas, J. J. Saenz, R. Gomez-Medina, and L. Chantada, Optical Forces on Small Magnetodielectric Particles, Opt. Express 18, 11428 (2010).

[25] A. Y. Bekshaev, Subwavelength Particles in an Inhomogeneous Light Field: Optical Forces Associated with the Spin and Orbital Energy Flows, J. Opt. 15, 044004 (2013).

[26] A. Canaguier-Durand, A. Cuche, C. Genet, and T. W. Ebbesen, Force and Torque on an Electric Dipole by Spinning Light Fields, Phys. Rev. A 88, 033831 (2013).

[27] K. Y. Bliokh, Y. S. Kivshar, and F. Nori, Magnetoelectric Effects in Local Light-Matter Interactions, Phys. Rev. Lett. 113, 033601 (2014).

[28] R. M. A. Azzam and N. M. Bashara, Ellipsometry and Polarized Light (North-Holland, Amsterdam, 1977).

[29] See Supplemental Material at http://link.aps.org/ supplemental/10.1103/PhysRevX.5.011039 for distributions of the interference wave fields with different polarizations, dynamical characteristics of the interference field with arbitrary polarizations of the two waves, and a detailed analysis of all the optical force and torque components for different polarizations of the field.

[30] K. Y. Bliokh, A. Y. Bekshaev, and F. Nori, Dual Electromagnetism: Helicity, Spin, Momentum, and Angular Momentum, New J. Phys. 15, 033026 (2013).

[31] A. Bekshaev, K. Y. Bliokh, and M. Soskin, Internal Flows and Energy Circulation in Light Beams, J. Opt. 13, 053001 (2011).

[32] S. Kocsis, B. Braverman, S. Ravets, M. J. Stevens, R. P. Mirin, L. K. Shalm, and A. M. Steinberg, Observing the Average Trajectories of Single Photons in a Two-Slit Interferometer, Science 332, 1170 (2011).

[33] D. E. Soper, Classical Field Theory (Wiley, New York, 1976).

[34] F. J. Belinfante, On the Current and the Density of the Electric Charge, the Energy, the Linear Momentum and the Angular Momentum of Arbitrary Fields, Physica (Amsterdam) 7, 449 (1940).

[35] H. C. Ohanian, What is Spin?, Am. J. Phys. 54, 500 (1986).

[36] K. Mita, Virtual Probability Current Associated with the Spin, Am. J. Phys. 68, 259 (2000).

[37] P. Banzer, M. Neugebauer, A. Aiello, C. Marquardt, N. Lindlein, T. Bauer, and G. Leuchs, The Photonic Wheel-Demonstration of a State of Light with Purely Transverse Angular Momentum, J. Eur. Opt. Soc. 8, 13032 (2013).

[38] M. R. Dennis and J. B. Gotte, Beam Shifts for Pairs of Plane Waves, J. Opt. 15, 014015 (2013).

[39] S. Sukhov, V. Kajorndejnukul, J. Broky, and A. Dogariu, Forces in Aharonov-Bohm Optical Setting, Optica 1, 383 (2014).

[40] M. Neugebauer, T. Bauer, A. Aiello, and P. Banzer, Measuring the Transverse Spin Density of Light, Phys. Rev. Lett. 114, 063901 (2015).

[41] C. F. Bohren and D. R. Huffman, Absorption and Scattering of Light by Small Particles (Wiley, New York, 1983). 